\title{
Evidence for a breakdown of the isobaric multiplet mass equation: A study of the $A=35, T=3 / 2$ isospin quartet
}

\author{
C. Yazidjian, ${ }^{1, *}$ G. Audi, ${ }^{2}$ D. Beck, ${ }^{1}$ K. Blaum, ${ }^{1,3}$ S. George, ${ }^{1,3}$ C. Guénaut,,${ }^{2,}$ F. Herfurth, ${ }^{1}$ A. Herlert, ${ }^{4, \ddagger}$ A. Kellerbauer,${ }^{5,}$ \\ H.-J. Kluge, ${ }^{1,6}$ D. Lunney, ${ }^{2}$ and L. Schweikhard ${ }^{4}$ \\ ${ }^{1}$ GSI, Planckstraße 1, D-64291 Darmstadt, Germany \\ ${ }^{2}$ CSNSM-IN2P3-CNRS, F-91405 Orsay-Campus, France \\ ${ }^{3}$ Institut für Physik, Johannes Gutenberg-Universität, D-55128 Mainz, Germany \\ ${ }^{4}$ Institut für Physik, Ernst-Moritz-Arndt-Universität, D-17487 Greifswald, Germany \\ ${ }^{5}$ CERN, Physics Department, CH-1211 Geneva 23, Switzerland \\ ${ }^{6}$ Ruprecht-Karls-Universität, Institut für Physik, D-69120 Heidelberg, Germany
}

(Received 3 May 2007; published 8 August 2007)

\begin{abstract}
Mass measurements on radionuclides along the potassium isotope chain have been performed with the ISOLTRAP Penning trap mass spectrometer. For ${ }^{35} \mathrm{~K}\left(T_{1 / 2}=178 \mathrm{~ms}\right)$ to ${ }^{46} \mathrm{~K}\left(T_{1 / 2}=105 \mathrm{~s}\right)$ relative mass uncertainties of $2 \times 10^{-8}$ and better have been achieved. The accurate mass determination of ${ }^{35} \mathrm{~K}(\delta \mathrm{m}=$ $0.54 \mathrm{keV}$ ) has been exploited to test the isobaric multiplet mass equation for the $A=35, T=3 / 2$ isospin quartet. The experimental results indicate a deviation from the generally adopted quadratic form.
\end{abstract}

DOI: 10.1103/PhysRevC.76.024308

\section{INTRODUCTION}

The application of the isospin formalism in nuclear physics stems from the assumption that the strong interaction is almost charge independent. In addition to the approximation that the neutron and the proton have the same mass, the isospin formalism describes the neutron and the proton as identical particles with isospin $T=1 / 2$ with the projections $T_{\mathrm{z}}(n)=+1 / 2$ and $T_{\mathrm{z}}(p)=-1 / 2$, respectively [1,2]. Isobaric nuclei with the same isospin $T$ belong to a $2 T+1$ multiplet with the projections $T_{\mathrm{z}}=(N-Z) / 2$, where $N$ is the number of neutrons and $Z$ the number of protons in the nucleus. The corresponding states of these isobars with the same $J^{\pi}$ and isospin $T$ are called isobaric analog states (IAS). The IAS have nearly the same wave function, mainly perturbed by the charge difference in the nuclei. By assuming only a two-body Coulomb force for the perturbation, the energy shift resulting from the charge-state difference can be calculated. In first-order perturbation theory, this approximation leads to a quadratic equation $[3,4]$

$$
D\left(A, T, T_{\mathrm{z}}\right)=a(A, T)+b(A, T) T_{\mathrm{z}}+c(A, T) T_{\mathrm{z}}^{2},
$$

which gives the mass excess $D$ of a member of a multiplet as a function of its atomic mass number $A$, its isospin $T$, and isospin projection $T_{\mathrm{z}}$. This relation is known as the isobaric multiplet mass equation (IMME).

${ }^{*}$ Corresponding address: CERN, Physics Department, CH-1211 Geneva 23, Switzerland; chabouh.yazidjian@cern.ch. This publication comprises part of the Ph.D. thesis of C. Yazidjian.

${ }^{\dagger}$ Present address: NSCL, Michigan State University, East Lansing, MI 48824-1321, USA.

${ }^{\ddagger}$ Present address: CERN, Physics Department, CH-1211 Geneva 23, Switzerland.

§Present address: Max Planck Institute for Nuclear Physics, P.O. Box 103980, D-69029 Heidelberg, Germany.
PACS number(s): 21.10.Dr, 07.75.+h, 21.60.Fw, 27.30.+t

The IMME can be used as a local mass model to predict unknown masses where some members of a multiplet are known. Short-range predictions can provide accurate masses with uncertainties as low as a few thousands of electron volts in favorable cases [5]. This can be very helpful for applications such as nuclear astrophysics, in particular, for modeling the rapid proton-capture (rp) process where such local models are in fact preferred to global models [6].

The IMME has been thoroughly studied in the late 1970s [7]. Since then, many additional measurements and tests have been performed and reported (see, e.g., Refs. [8-10]). The IMME was found to work very well for most cases. However, from the latest data compilation [11] and recent results of experiments, some cases show a deviation from the quadratic form of the IMME and need additional higher order terms [12]. Tests require systems with at least four nuclides in the multiplet (i.e., with an isospin $T \geqslant 3 / 2$ ). Up to now, only the $A=9, T=3 / 2$ quartet and the $A=8, T=2$ quintet system are known to deviate significantly (i.e., by more than three standard deviations) from the quadratic form of the IMME (see Fig. 1). For those multiplets higher order terms have to be added, either $d T_{\mathrm{z}}^{3}$ or $e T_{\mathrm{z}}^{4}$, or both. The present paper reports on the improvement of the ${ }^{35} \mathrm{~K}$ mass, which allows a further test of the quadratic form of the IMME for the $A=35, T=3 / 2$ isospin quartet.

\section{THE ISOLTRAP EXPERIMENT}

The tandem Penning trap mass spectrometer ISOLTRAP [13], installed at the on-line isotope separator ISOLDE [14] at CERN (Geneva), is an experiment dedicated to high-precision mass measurements on short-lived radionuclides $[15,16]$. The mass measurement is based on the determination of the cyclotron frequency $v_{\mathrm{c}}$ of a stored ion, probed by use of radiofrequency (rf) fields in a Penning trap. With a charge-tomass ratio $q / m$ and a magnetic field $B$ the cyclotron frequency 


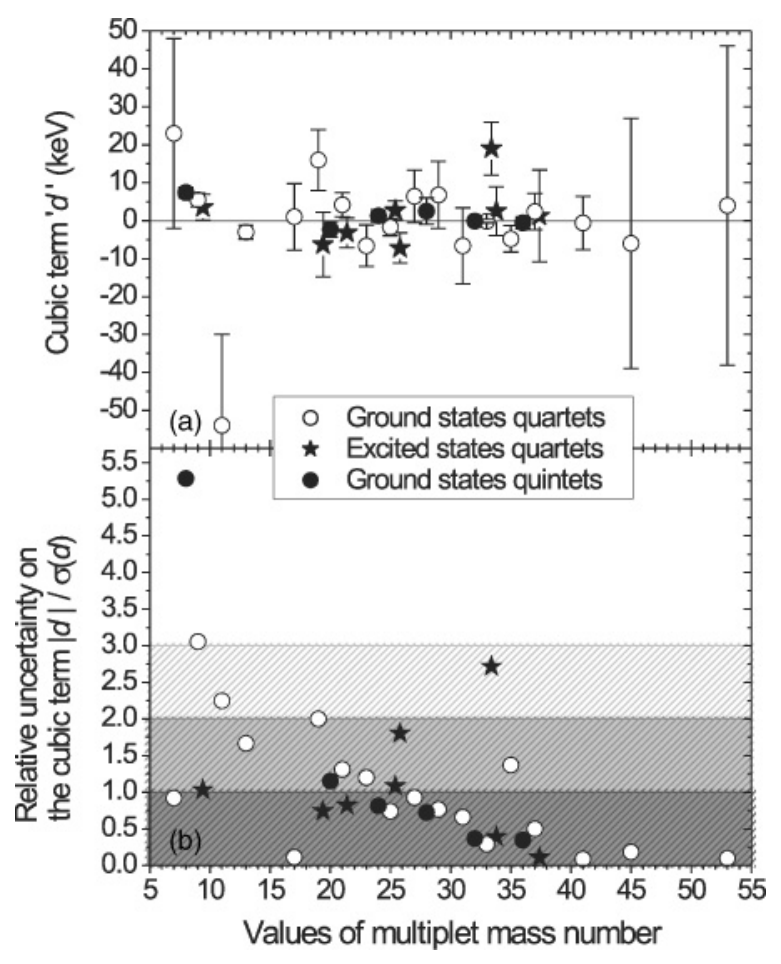

FIG. 1. Compilation for the cubic parameter $d$ of the IMME [8-11]. Top: Values of $d$ as a function of the mass value $A$. Bottom: Relative uncertainty of $d$ where the shaded areas indicate the $1 \sigma, 2 \sigma$, and $3 \sigma$ agreement.

is given by

$$
v_{\mathrm{c}}=\frac{1}{2 \pi} \frac{q}{m} B .
$$

With this technique, a relative mass uncertainty of the order of $\delta m / m=10^{-8}$ is routinely reached with ISOLTRAP [17].

The setup is composed mainly of three parts (see Fig. 2): First, a linear radiofrequency quadrupole (RFQ) cooler and buncher has the task to stop, accumulate, cool, and bunch the 60-keV ISOLDE beam for a subsequent transfer into the preparation trap [18]. Second, the cylindrical preparation Penning trap with helium buffer gas is used to remove isobaric contaminants [19] with a resolving power $R=m / \Delta m$ up to $10^{5}$ and to bunch the ions for an efficient delivery to the second Penning trap. Finally, in the hyperbolical precision Penning trap the cyclotron frequency $v_{\mathrm{c}}$ is determined for both the ion of interest and a well-known reference ion by use of a quadrupolar rf excitation [20], for which the frequency is varied around $v_{\mathrm{c}}$. The duration of the $\mathrm{rf}$ excitation is chosen between 0.1 and $9 \mathrm{~s}$ depending on the required resolution and the half-life of the ion of interest. As an example of the time-of-fight technique [21] used at ISOLTRAP, the inset in Fig. 2 shows a cyclotron resonance for ${ }^{35} \mathrm{~K}^{+}$.

For the production of radioactive potassium isotopes a titanium-foil target was used. It consists of a stack of thin titanium foils ( $30 \mu \mathrm{m}$ each) for a total thickness of $19 \mathrm{~g} \mathrm{~cm}^{-2}$ and a quantity of $50 \mathrm{~g}$ of titanium. The radioactive potassium isotopes were produced by bombardment with $1.4-\mathrm{GeV}$ protons from the CERN Proton-Synchrotron Booster on the ISOLDE target. After diffusing out of the heated target they
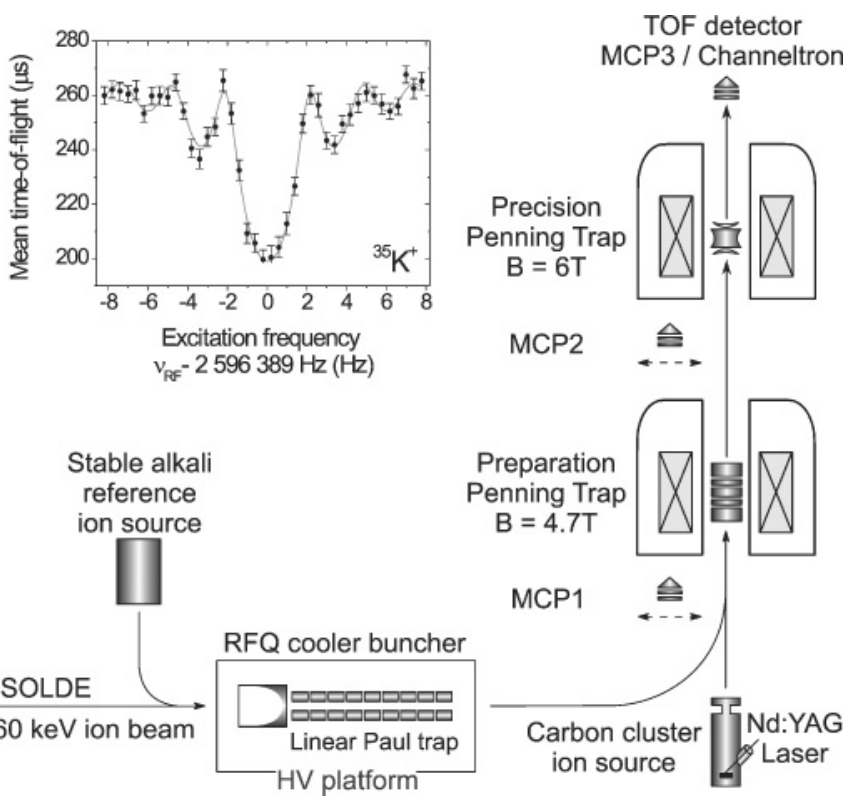

FIG. 2. Sketch of the experimental setup of the ISOLTRAP mass spectrometer. Microchannel plate (MCP) detectors are used to monitor the ion-beam transfer (MCP1-2). A newly implemented channeltron detector [22], which can be used in place of MCP3, records the time-of-flight (TOF) resonance curve for the cyclotron frequency determination. The inset shows a resonance curve for ${ }^{35} \mathrm{~K}^{+}$(with excitation duration of $400 \mathrm{~ms}$ ) together with the fit of the theoretically expected line shape to the data points [23].

were surface-ionized by a hot tungsten ionizer. The potassium ions were then accelerated to $60 \mathrm{keV}$ and delivered to the ISOLTRAP experiment via the ISOLDE High-Resolution Separator (HRS). During three days, the isotopic chain of potassium has been explored from ${ }^{35} \mathrm{~K}$ for the neutron-deficient side up to ${ }^{46} \mathrm{~K}$ for the neutron-rich side. A total number of 29 cyclotron resonances for radioactive potassium isotopes have been recorded, together with 39 resonances of the stable reference ion ${ }^{39} \mathrm{~K}^{+}$. No ion contamination was observed during the beam time. In the following the ISOLTRAP mass values of the potassium isotopes are discussed, especially the ${ }^{35} \mathrm{~K}$ mass and its influence on the quadratic form of the IMME.

\section{RESULTS}

Table I summarizes the measured frequency ratios and the deduced mass-excess values of the investigated isotopes. The mass determination for the potassium isotopes ${ }^{35} \mathrm{~K}\left(T_{1 / 2}=\right.$ $178 \mathrm{~ms})$ up to ${ }^{46} \mathrm{~K}\left(T_{1 / 2}=105 \mathrm{~s}\right)$ has been performed with an uncertainty below $2 \times 10^{-8}$, that is, a reduction of the mass uncertainty by a factor of up to 40 for the neutron-deficient side $\left({ }^{35} \mathrm{~K}\right)$ and close to 80 for the neutron-rich potassium isotopes $\left({ }^{44} \mathrm{~K}\right)$ as compared to the literature values [24,25]. An overview is shown in Fig. 3. The data for the well-known isotopes ${ }^{37-39} \mathrm{~K}$ are plotted as a cross-check for the measurement process and the reliability of the ISOLTRAP results.

The mass excess for ${ }^{35} \mathrm{~K}$ found in this work $\left[D_{\exp }=\right.$ $-11172.73(54) \mathrm{keV}$ ] agrees with the value given in the latest Atomic-Mass Evaluation (AME2003) [24] but has a 40 times 
TABLE I. Frequency ratios relative to the ${ }^{39} \mathrm{~K}^{+}$reference ion and mass-excess values of the investigated potassium isotopes. The mass excess $D_{\text {exp }}$ is calculated by $D_{\exp }=M_{\exp }-A$, where $A$ is the respective mass number and $M_{\exp }$ the atomic mass as deduced from the experimentally determined frequency ratio: $M_{\mathrm{exp}}=\left(M^{\mathrm{ref}}-m_{\mathrm{e}}\right) v_{\mathrm{c}}^{\mathrm{ref}} / v_{\mathrm{c}}+m_{\mathrm{e}}$.

\begin{tabular}{lcccrr}
\hline \hline Isotope & $T_{1 / 2}{ }^{\mathrm{a}}$ & Frequency ratio $^{\mathrm{b}} \nu_{\mathrm{c}}^{\text {ref }} / \nu_{\mathrm{c}}$ & $D_{\exp }{ }^{\mathrm{c}}(\mathrm{keV})$ & $D_{\text {lit }}{ }^{\mathrm{a}}(\mathrm{keV})$ & $\Delta=D_{\text {lit }}-D_{\exp }(\mathrm{keV})$ \\
\hline${ }^{35} \mathrm{~K}$ & $178(8) \mathrm{ms}$ & $0.8979625551(140)$ & $-11172.73(54)$ & $-11169(20)$ & 3.7 \\
${ }^{36} \mathrm{~K}$ & $342(2) \mathrm{ms}$ & $0.9234557832(97)$ & $-17416.83(39)$ & $-17426(8)$ & -9.2 \\
${ }^{37} \mathrm{~K}$ & $1.22(1) \mathrm{s}$ & $0.9489176146(84)$ & $-24800.45(35)$ & $-24800.20(9)$ & 0.3 \\
${ }^{38} \mathrm{~K}$ & $7.64(2) \mathrm{min}$ & $0.9744726675(112)$ & $-28800.69(45)$ & $-28800.7(4)$ & 0.0 \\
${ }^{43} \mathrm{~K}$ & $22.3(1) \mathrm{h}$ & $1.1025848117(113)$ & $-36575.19(46)$ & $-36593(9)$ & -17.9 \\
${ }^{44} \mathrm{~K}$ & $22.1(2) \mathrm{min}$ & $1.1282719566(115)$ & $-35781.29(47)$ & $-35810(40)$ & -28.8 \\
${ }^{45} \mathrm{~K}$ & $17.3(6) \mathrm{min}$ & $1.1539142443(144)$ & $-36615.43(57)$ & $-36608(10)$ & -4.3 \\
${ }^{46} \mathrm{~K}$ & $105(10) \mathrm{s}$ & $1.1796126258(201)$ & $-35413.71(76)$ & $-35418(16)$ & \\
\hline \hline
\end{tabular}

${ }^{a}$ Values from Ref. [24].

${ }^{\mathrm{b}}$ Using ${ }^{39} \mathrm{~K}^{+}$as a reference.

${ }^{\mathrm{c}} M^{\mathrm{ref}}\left({ }^{39} \mathrm{~K}\right)=38963706.68(20) \mu \mathrm{u}$ [25], $m_{\mathrm{e}}=548579.9110$ (12) nu [26], and $1 \mathrm{u}=931494.009(7) \mathrm{keV}$ [26].

smaller uncertainty. The consequences with respect to the quadratic form of the IMME are discussed in detail in the next section.

For ${ }^{36} \mathrm{~K}$, with a mass excess of $D_{\exp }=$ -17416.83 (39) $\mathrm{keV}$, the only contribution to the AME2003 arises from the ${ }^{36} \operatorname{Ar}(p, n)^{36} \mathrm{~K}$ reaction [27] and gives originally $D=-17421(8) \mathrm{keV}$. It has to be emphasized that the $(p, n)$ reaction energy has been recalibrated afterward [28]. Other indirect mass determinations from ${ }^{36} \operatorname{Ar}(p, n)^{36} \mathrm{~K}$ [29] and ${ }^{36} \mathrm{Ar}\left({ }^{3} \mathrm{He}, t\right){ }^{36} \mathrm{~K}$ [30] can also be found in the literature but were not used for the mass evaluation. Whereas the value given in Ref. [29] agrees within the uncertainty, a deviation of $2.4 \sigma$ is observed relative to the value reported in Ref. [30]. Finally, only a slight difference is observed compared to the literature value from the recalibrated experiment of Goosman et al.: $D_{\text {lit }}=-17426.2(7.8) \mathrm{keV}$ [24] with $1.1 \sigma$. The mass of ${ }^{36} \mathrm{~K}$ also has an impact on the IMME test for the $A=36, T=2$ quintet. The consequences for the

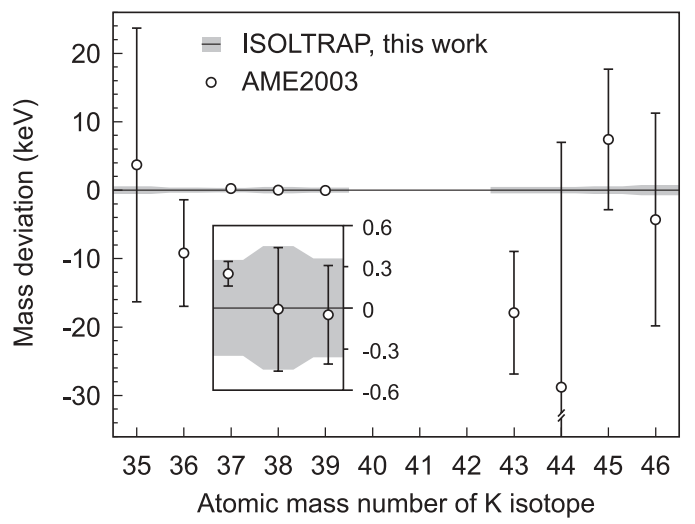

FIG. 3. Difference of mass-excess values $\left(D_{\text {lit }}-D_{\text {exp }}\right)$ of the potassium isotopes taken from this work $\left(D_{\text {exp }}\right.$; see Table I) and the literature $[24,25]$. The experimental value of ${ }^{39} \mathrm{~K}$ results from a consistency check of the ISOLTRAP data (see text). The shaded area represents the uncertainty of the ISOLTRAP values. The differences of the mass-excess values of ${ }^{37-39} \mathrm{~K}$ are given as a cross-check. For more details refer to the discussion section. quadratic form of the IMME are presented together with the $A=35, T=3 / 2$ quartet in Sec. IV.

The ISOLTRAP value for the mass excess of ${ }^{37} \mathrm{~K}\left[D_{\exp }=\right.$ $-24800.45(35) \mathrm{keV}$ ] has four times the uncertainty of the literature value, since the latter is known with a precision better than the current limit of our experiment $\sigma(m) / m=8 \times 10^{-9}$. However, the present result shows an excellent agreement with the adopted mass-excess value, which gives strong confidence in the ISOLTRAP results.

For ${ }^{38} \mathrm{~K}$ an isomeric state might have been present during the measurements. The excitation energy of the isomeric state, $E^{*}=130.4$ (3) $\mathrm{keV}$, is well determined [31] by measurements of the ${ }^{38} \mathrm{~K}^{m}(\mathrm{IT}){ }^{38} \mathrm{~K}$ internal transition $\gamma$ rays, after the production of ${ }^{38} \mathrm{~K}^{m}$ with a ${ }^{38} \operatorname{Ar}(p, n)^{38} \mathrm{~K}^{m}$ reaction. In specific radioactive-beam preparation and together with laser ionization using the resonant laser ionization method RILIS [32], the ISOLTRAP experiment showed its ability to perform pure isomeric mass determination in the case of ${ }^{68} \mathrm{Cu}$ [33] and ${ }^{70} \mathrm{Cu}$ [34]. With an excitation time of $T_{\mathrm{RF}}=900 \mathrm{~ms}$, the resolving power was about $2 \times 10^{6}$ (i.e., one order of magnitude higher than needed to resolve the two respective isomers). However, in the present work only one of the isomeric states has been observed. For this reason, the resulting mass could not be clearly assigned directly to any of the two isomeric states. The excited state ${ }^{38} \mathrm{~K}^{m}$ is shorter lived $(924 \mathrm{~ms})$ than the ground state ${ }^{38} \mathrm{~K}(7.64 \mathrm{~min})$. During the cyclotron frequency determination procedure, radioactive nuclides may decay and produce characteristic signals in the time-of-flight spectrum. The analysis of the cyclotron-resonance data performed with an excitation time of $1.2 \mathrm{~s}$ did, however, not show any decay peaks. Moreover, the obtained mass-excess value -28800.69 (45) $\mathrm{keV}$ agrees with the literature value of the ground state: -28800.7 (4) $\mathrm{keV}$ [24]. Therefore it can be concluded that the ground state ${ }^{38} \mathrm{~K}$ and not the excited isomeric state was produced in the ISOLDE target and directly measured with ISOLTRAP.

Even though ${ }^{39} \mathrm{~K}$ was used as the reference nuclide for all potassium measurements, all the backward information flow from the investigated nuclides provided a contribution to a new mass evaluation for ${ }^{39} \mathrm{~K}$. In Ref. [24], the mass evaluation from all experimental data is done by solving a 
TABLE II. Mass excess for nuclides of the $A=35, T=3 / 2$ quartet. The members of the $A=35$ quartet with their respective half-life and the associated isospin are given in the first three columns. The corresponding mass excess of the ground state and the excitation energy and their uncertainty for the IAS are given in columns 4 and 5. The last column indicates the total mass excess entering in the IMME.

\begin{tabular}{lccccc}
\hline \hline Nucleus & $T_{1 / 2}{ }^{\mathrm{a}}$ & \multicolumn{1}{c}{$T_{\mathrm{z}}$} & $D_{\exp }^{\mathrm{gs} a \mathrm{a}}(\mathrm{keV})$ & $E_{\exp }^{* \mathrm{~b}}$ & $D_{\exp }^{\text {tot }}(\mathrm{keV})$ \\
\hline${ }^{35} \mathrm{~K}$ & $178(8) \mathrm{ms}$ & $-3 / 2$ & $-11172.73(0.54)^{\mathrm{c}}$ & - & $-11172.73(0.54)$ \\
${ }^{35} \mathrm{Ar}$ & $1.775(4) \mathrm{s}$ & $-1 / 2$ & $-23047.41(0.75)$ & $5572.71(0.17)$ & $-17474.70(0.77)$ \\
${ }^{35} \mathrm{Cl}$ & stable & $1 / 2$ & $-29013.54(0.04)$ & $5654(2)$ & $-23359.54(2.00)$ \\
${ }^{35} \mathrm{~S}$ & $87.51(12) \mathrm{d}$ & $3 / 2$ & $-28846.36(0.10)$ & - & $-28846.36(0.10)$ \\
\hline \hline
\end{tabular}

${ }^{\mathrm{a}}$ Ground state; values from Ref. [24].

${ }^{\mathrm{b}}$ Values from Ref. [11].

${ }^{\mathrm{c}}$ This work.

system of linear equations. In the present work, the mass determination of the well-known nuclides ${ }^{37} \mathrm{~K}$ and ${ }^{38} \mathrm{~K}$, used for cross-references and data consistency, slightly changed the ${ }^{39} \mathrm{~K}$ mass-excess value $\left[D_{\text {exp }+ \text { lit }}=-33806.9\right.$ (2) keV, instead of $D_{\text {lit }}=-33807.0(2) \mathrm{keV}$ ]. This is of interest since ${ }^{39} \mathrm{~K}$ is used as a reference mass in many experimental setups.

Previous work $[35,36]$ led to two different mass values for ${ }^{43} \mathrm{~K}$. The adopted value in Ref. [24], which results from an average of those input data, has a large uncertainty $\left[D_{\text {lit }}\left({ }^{43} \mathrm{~K}\right)=-36593(9) \mathrm{keV}\right]$. The value presented in this work shows a relative discrepancy of a bit more than two standard deviations. The adopted literature value results from indirect mass determinations. Sometimes such a deviation can be explained by missing $\gamma$ lines in the recorded spectra. However, in the present case, no clear indication of missing levels has been found.

Former investigations on the decay imply large uncertainties of the order of $10 \mathrm{keV}$ for ${ }^{44} \mathrm{~K}$. Comparison between the mass-excess value presented in this work and the original data shows good agreement with results from Ref. [37] and a deviation of $1.3 \sigma$ from Ref. [38]. However, the weightedaverage mass-excess value given in Ref. [24] agrees with the ISOLTRAP data.
The uncertainties in ${ }^{45} \mathrm{~K}$ and ${ }^{46} \mathrm{~K}$ arise from the reactions ${ }^{46} \mathrm{Ca}(t, \alpha){ }^{45} \mathrm{~K}[39]$ and ${ }^{48} \mathrm{Ca}(d, \alpha){ }^{46} \mathrm{~K}[40]$ but those articles are not well documented for a recalibration of the measurements (see Ref. [26] and references therein). The mass-excess values presented in this work agree with the previous data and improve the uncertainties by a factor of 20 to 80 .

\section{DISCUSSION}

The two potassium isotopes ${ }^{35} \mathrm{~K}$ and ${ }^{36} \mathrm{~K}$ are involved in the $A=35, T=3 / 2$ isospin quartet and the $A=36, T=2$ isospin quintet, respectively. In Tables II and III, the updated mass-excess values of the multiplets are summarized by taking into account the values presented in this work. Unfortunately, to fully evaluate the $A=36$ quintet requires a precise mass determination of ${ }^{36} \mathrm{Ca}\left[T_{1 / 2}=102(2) \mathrm{ms}\right]$, which is still lacking $\left[D_{\text {lit }}=-6440(40) \mathrm{keV}\right]$.

From the mass-excess values, the coefficients $a, b$, and $c$ of the quadratic terms and the possible coefficient $d$ of the cubic term of the IMME can be derived by use of a standard least mean square fit. The results for the $A=35, T=3 / 2$ quartet, and for the $A=36, T=2$ quintet, under the assumption of quadratic and cubic forms of the IMME, are given in Tables IV and $\mathrm{V}$.

TABLE III. Mass excess for nuclides of the $A=36, T=2$ quintet. The members of the $A=36$ quintet with their respective half-life and the associated isospin are given in the first three columns. The corresponding mass excess of the ground state and the excitation energy and their uncertainty for the IAS are given in columns 4 and 5. The last column indicates the total mass excess entering in the IMME.

\begin{tabular}{lcrlcc}
\hline \hline Nucleus & $T_{1 / 2}{ }^{\mathrm{a}}$ & $T_{\mathrm{z}}$ & $D_{\exp }^{\mathrm{gs} a}(\mathrm{keV})$ & $E_{\exp }^{*}(\mathrm{keV})$ & $D_{\exp }^{\mathrm{tot}}(\mathrm{keV})$ \\
\hline${ }^{36} \mathrm{Ca}$ & $102(2) \mathrm{ms}$ & -2 & $-6440(40)$ & - & $-6440(40)$ \\
${ }^{36} \mathrm{~K}$ & $142(2) \mathrm{ms}$ & -1 & $-17416.83(0.39)^{\mathrm{c}}$ & $4282.2(2.5)^{\mathrm{d}}$ & $-13134.7(2.4)^{\mathrm{e}}$ \\
${ }^{36} \mathrm{Ar}$ & stable & 0 & $-30231.54(0.03)^{\mathrm{f}}$ & $10851.6(1.5)$ & $-19379.94(1.50)$ \\
${ }^{36} \mathrm{Cl}$ & $301(2) \mathrm{ky}$ & 1 & $-29521.86(0.07)$ & $4299.70(0.08)$ & $-25222.16(0.11)$ \\
${ }^{36} \mathrm{~S}$ & stable & 2 & $-30664.07(0.19)$ & - & $-30664.07(0.19)$ \\
\hline \hline
\end{tabular}

a Ground state; values from Ref. [24].

${ }^{b}$ Values from Refs. [11,31,41].

${ }^{\mathrm{c}}$ This work.

${ }^{\mathrm{d}}$ Extracted from the ground-state and the excited-state values (see footnote ${ }^{\mathrm{e}}$ ).

${ }^{\text {e}}$ Value from Ref. [42] corrected for relativistic effect and for the ${ }^{35} \mathrm{Ar}$ mass [24].

${ }^{\mathrm{f}} \mathrm{See}$ Sec. 7.4 in Ref. [26]. 
TABLE IV. The $A=35, T=3 / 2$ quartet and the coefficients for the quadratic and cubic forms.

\begin{tabular}{lccccc}
\hline \hline$D\left(T_{\mathrm{z}}\right)$ & $a(\mathrm{keV})$ & $b(\mathrm{keV})$ & $c(\mathrm{keV})$ & $d(\mathrm{keV})$ & $\chi_{n}^{2}$ \\
\hline Quadratic & $-20470.7(0.8)$ & $-5891.2(0.2)$ & $205.0(0.4)$ & - & 8.8 \\
Cubic & $-20468.1(0.2)$ & $-5884.0(2.4)$ & $203.8(0.6)$ & $-3.2(1.1)$ & - \\
\hline \hline
\end{tabular}

The updated plot for the cubic term $d$ of the IMME is given in Fig. 4. In the latest compilation [11], the $A=35, T=$ $3 / 2$ isospin quartet was already reported to slightly deviate, and the $A=36$ quintet followed the adopted quadratic form with a cubic term with the coefficient $d=-0.6(1.6) \mathrm{keV}$. In Ref. [11] the reduced $\chi^{2}$ for the quintet was close to 3 , therefore indicating a possible inconsistent set of data.

However, it can be observed that for the $A=35$ quartet none of the coefficients of the quadratic form agrees within one standard deviation with the corresponding coefficient for the cubic form. This clearly indicates a strong discrepancy and is an argument in favor of using higher terms to describe the IMME. For the $A=36$ quintet, which now shows no deviation within a standard deviation, the uncertainties on the coefficients are larger, because of the lack of knowledge on the ${ }^{36} \mathrm{Ca}$ ground state. Unless a new high-precision mass measurement is performed, no final conclusions about the quintet can be drawn. However, if one assumes the quadratic form of the IMME to be valid for the $A=36, T=2$ quintet, the mass value of ${ }^{36} \mathrm{Ca}$ can be extrapolated from the other members of the multiplet to $D_{\mathrm{IMME}}=-6490.3(6) \mathrm{keV}$. This value slightly deviates $(1.3 \sigma)$ from the previously adopted value [24] but has a close to two orders of magnitude smaller uncertainty.

To understand the reasons for the deviation observed for the $A=35$ quartet and to find out the causes for higher order terms in the IMME, it will be assumed that the quadratic form of the IMME is correct and that one (or more) of the ground-state masses involved exhibit a systematic shift. The same signature appears if one of the excited states is wrongly assigned.

Table VI shows the different mass-excess predictions of the supposedly "unknown" nuclides, calculated with the fit parameters given in Table IV. The ${ }^{35} \mathrm{~K},{ }^{35} \mathrm{Ar}$, and ${ }^{35} \mathrm{~S}$ values agree with those in the literature. However, compared to the value given in Refs. $[11,24]$ the ${ }^{35} \mathrm{Cl}$ mass deviates by 2.6 standard deviations. Owing to the $2 \mathrm{keV}$ uncertainty on the IAS, the $T_{\mathrm{z}}=1 / 2$ member state in ${ }^{35} \mathrm{Cl}$ has the least significant contribution to the fit. The present status is identical to the IMME "breakdown" reported in Ref. [8], where the least significant member was ${ }^{33} \mathrm{Ar}$. The "revalidation" of the IMME [9] showed that the excited state of ${ }^{33} \mathrm{Cl}$ was erroneous. Therefore, even if those direct mass extrapolation methods seem to indicate a deviation of the excited $T_{\mathrm{z}}=1 / 2$ isobaric analog state for ${ }^{35} \mathrm{Cl}$, caution is advised.

In the following, a more detailed discussion is presented, where all ground-state mass values for the members of the $A=35, T=3 / 2$ quartet as well as the excitation energies for ${ }^{35} \mathrm{Cl}$ and ${ }^{35} \mathrm{Ar}$ are included to find indications for any deviation of the IMME.

\section{A. Ground-state masses of the IMME $A=35$ quartet}

In the $A=35, T=3 / 2$ quartet four ground-state masses are involved: In this work the mass excess of ${ }^{35} \mathrm{~K}$ has been directly determined for the first time by a Penning trap measurement technique. Only few cases showed a discrepancy to the Atomic-Mass Evaluation [24] that could not be resolved, as, for example, ${ }^{36} \mathrm{Ar}$ (see Sec. 7.4 of Ref. [26] and references therein). Moreover, the precision of the mass-excess value in this work is 40 times better than the adopted one and agrees with it.

The mass excess of ${ }^{35} \mathrm{Ar}$ results from an indirect mass measurement by means of the ${ }^{35} \mathrm{Cl}(p, n){ }^{35} \mathrm{Ar}$ reaction. Three input data values are taken [43-45]. However, one of them [45] deviates by $3.4 \mathrm{keV}$ (close to two standard deviations) from the adopted value. Nevertheless, this deviation alone is not sufficient to explain the discrepancy of the quadratic form of the IMME.

The mass of ${ }^{35} \mathrm{Cl}$ has been determined by direct $\mathrm{rf}$ measurements [46], which contribute about $79 \%$ to the mass determination. Since the value stems from a direct measurement, it is quite reliable and can be assumed to be correct.

The mass excess of ${ }^{35} \mathrm{~S}$ is mainly determined $(95 \%)$ by a $\beta$-endpoint measurement of the ${ }^{35} \mathrm{~S}\left(\beta^{-}\right){ }^{35} \mathrm{Cl}$ reaction, which was thoroughly studied for the presumed existence of a 17-keV neutrino (see Ref. [26] Sec. 7.3 and references therein). Even though the data reported in Ref. [26] are labeled as "well documented but not consistent with other well documented data," the discrepancies observed are less than $0.4 \mathrm{keV}$ [47-52]. Although those relatively small uncertainties and deviations from the adopted value are not sufficient to draw conclusions on the existence or absence of the $17-\mathrm{keV}$

TABLE V. The $A=36, T=2$ quintet and the coefficients for the quadratic and cubic forms.

\begin{tabular}{lccccc}
\hline \hline$D\left(T_{\mathrm{z}}\right)$ & $a(\mathrm{keV})$ & $b(\mathrm{keV})$ & $c(\mathrm{keV})$ & $d(\mathrm{keV})$ & $\chi_{n}^{2}$ \\
\hline Quadratic & $-19379.1(0.7)$ & $-6043.6(0.8)$ & $200.6(0.3)$ & - & 0.9 \\
Cubic & $-19380.3(1.5)$ & $-6043.3(1.1)$ & $202.1(1.8)$ & $-0.7(0.8)$ & 1.1 \\
\hline \hline
\end{tabular}


TABLE VI. Mass prediction and residuals found by assuming a quadratic fit and using the coefficients as given in Table IV.

\begin{tabular}{lcrc}
\hline \hline \multicolumn{4}{c}{$D\left(T_{\mathrm{z}}\right)=a+b T_{\mathrm{z}}+c T_{\mathrm{z}}^{2}$} \\
\hline Nucleus & $D_{\text {cal }}^{\text {tot }}(\mathrm{keV})$ & $D_{\text {cal }}^{\text {tot }}-D_{\text {exp }}^{\text {tot }}(\mathrm{keV})$ & $E_{\text {cal }}^{*}(\mathrm{keV})$ \\
\hline${ }^{35} \mathrm{~K}$ & $-11172.9(1.2)$ & $-0.2(1.3)$ & - \\
${ }^{35} \mathrm{Ar}$ & $-17473.9(0.8)$ & $0.8(1.1)$ & $5573.5(1.1)$ \\
${ }^{35} \mathrm{Cl}$ & $-23365.0(0.8)$ & $-5.5(2.1)$ & $5648.5(0.8)$ \\
${ }^{35} \mathrm{~S}$ & $-28846.4(1.2)$ & $0.0(1.2)$ & - \\
\hline \hline
\end{tabular}

neutrino, they are precise enough to presume that the mass value of ${ }^{35} \mathrm{~S}$ is accurate, since no systematic trends were found in the literature.

Thus, the careful study of the ground states did not show any deviation from the adopted values [24], except maybe for ${ }^{35} \mathrm{Ar}$. In Ref. [26], this nuclide is labeled as "secondary data," that is, where the mass is known from only one type of data; in the present case the experimental input is from the ${ }^{35} \mathrm{Cl}(p, n){ }^{35} \mathrm{Ar}$ reaction [43-45] and is not cross-checked by a different connection.

\section{B. Excited states of the IMME $A=35$ quartet}

The values reported for the excited states are taken from Refs. $[11,25,31,41]$ and references therein. The adopted value for the excited state of ${ }^{35} \mathrm{Ar}$ does not show a strong deviation from the experimental data. In addition, the different estimates for the excited state in Table VI do not deviate by more than one standard deviation and are far from any other known excited state in ${ }^{35} \mathrm{Ar}$. Therefore, it can be concluded that the excited state is correctly assigned.

For the excited state of ${ }^{35} \mathrm{Cl}$, as summarized in Ref. [31], the experimental data are not precise enough and show as well some discrepancies (see Ref. [53] and references therein). The energy level scheme of ${ }^{35} \mathrm{Cl}$ exhibits a "double" peak around 5.65 MeV, which has been thoroughly investigated [53-57]. Previous work on the IMME showed that the energy of the excited state for ${ }^{33} \mathrm{Cl}$ was wrongly calculated from the center of mass to the laboratory frame [8]. From the raw data of

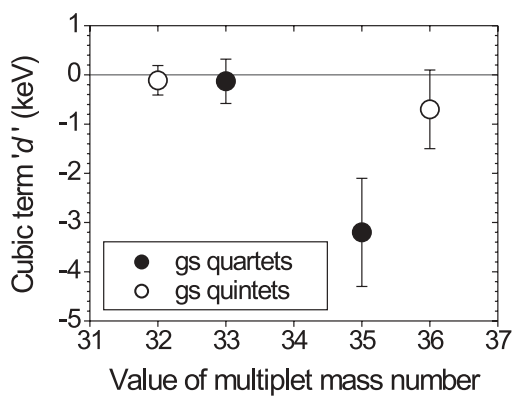

FIG. 4. Partially updated compilation of the coefficient $d$ of the cubic term for multiplets, including this work. The four points shown correspond to the $A=33$ and $A=35$ quartets, and to the $A=32$ and $A=36$ quintets for which at least one member has been measured at ISOLTRAP $[8,10]$. The two points on the right-hand side (i.e., the $A=35$ quartet and the $A=36$ quintet) arise from the present work. the proton energy in the laboratory frame [57], the excitation energy has been recalculated by taking into account relativistic effects and compared to the values given in the aforementioned references. No major deviation was found.

A detailed analysis of the excited state of ${ }^{35} \mathrm{Cl}$ and a discussion of the separation and the spin assignment of the two 5.65-MeV states can be found in Ref. [56]. The excitation energy resulting from the mean values of the data gives the respective energies of 5646 (2) and 5654 (2) $\mathrm{keV}$. The excited state at 5654 (2) $\mathrm{keV}$ is the commonly adopted value for the $T=3 / 2$ isobaric analog state. However, the calculated excitation energy as given in Table VI corresponds to the excited state with energy 5646 (2) $\mathrm{keV}$. When one uses this state rather than the adopted one, a cubic term $d=$ $0.8(1.0) \mathrm{keV}$ is found in agreement with zero. Therefore, it can be concluded that a misassignment of the isobaric analog state is possible.

Moreover, the $Q$ value found in Ref. [53] shows a deviation of about $3 \mathrm{keV}$ as compared to that in Ref. [24] for the ${ }^{34} \mathrm{~S}(p, \gamma){ }^{35} \mathrm{Cl}$ reaction. For the $A=33$ quartet [9] an unexpected shift of a few $\mathrm{keV}$ was revealed for the excited states of ${ }^{33} \mathrm{Cl}$. This shift was sufficient to explain the observed "breakdown" of the IMME [8] and revalidated the quadratic form of the IMME [9]. Such a trend can also be the source of the deviation for the $A=35, T=3 / 2$ quartet.

\section{Higher order terms of the IMME $A=35$ quartet}

In the discussion so far, it has been assumed that the IMME has a pure quadratic form and that an indication for a possibly wrong mass and/or excitation energy has been found. Even though the adopted quadratic form of the IMME is sufficient to describe the mass surface for a given multiplet, experimental and theoretical studies pointed out the possibility of a deviation from the quadratic form and the need for higher order terms in the IMME [12].

The excited-state assignment of ${ }^{35} \mathrm{Cl}$ can be validated with a simulation based on a theoretical model without isospin mixing [58]. The $3 / 2^{+}$excited state, corresponding to the isobaric analog state in the $A=35, T=3 / 2$ quartet, shows a preferential branching ratio toward the $5 / 2^{+}$state lying at $3 \mathrm{MeV}$. Compared to the decay scheme of bound states in ${ }^{35} \mathrm{Cl}$, where the 5654 (2) and 5646 (2) $\mathrm{keV}$ states decay toward the $5 / 2^{+}$and the $7 / 2^{-}$states, respectively, it can be concluded that there is no misassignment of the IAS in ${ }^{35} \mathrm{Cl}$. Calculations based on $s d$-shell-model calculations [59] with isospin-dependent interaction show as well a deviation from the quadratic form of the IMME with the same magnitude for the coefficient of the cubic term but with opposite sign (i.e., $d=3.1 \mathrm{keV}$ ). The reasons for the sign difference are not clear yet but the IMME quadratic form seems to be insufficient to describe the $A=35, T=3 / 2$ quartet from both the experimental and the theoretical sides.

The pure two-body Coulomb perturbation approximation to derive the IMME neglects the off-diagonal part of the isovector and isotensor components of the Coulomb force. However, the latter might introduce an isospin mixing, which causes a shift in the levels of the different quartet members and leads to a higher order polynomial form in $T_{\mathrm{z}}$. 
It has been demonstrated in Ref. [60] that corrections to the quadratic form of the IMME can be used. The correction with the coefficient $d$ of the cubic term is expected to be proportional to $Z \alpha c$, where $Z$ is the proton number, $\alpha$ the fine-structure constant, and $c$ the coefficient of the quadratic form of the IMME. However, the calculated $d$ values are found to be smaller than $Z \alpha c$. This can be explained by the fact that the second-order corrections are "absorbed" in the $a, b, c$ coefficients. Isospin violation of the nuclear interaction inducing a small isospin-breaking component can also lead to higher order terms in the IMME. If the bare nuclear interaction has a three-body component, and if it is isospin violating, it would automatically lead to a cubic $T_{z}^{3}$ term. In the vicinity of the $A=35, T=3 / 2$ quartet members unexpected isospin-breaking and isospin-mixing effects have been recently observed for the $7 / 2^{-}$and $13 / 2^{-}$states between the ${ }^{35} \mathrm{Cl}$ and ${ }^{35} \mathrm{Ar}$ mirror nuclides [61]. If the isospin $T$ is a good quantum number, the $E 1$ transitions are identical in mirror nuclei, which is not the case. The reason for this is an isospin mixing of the $\left|7 / 2^{-}\right\rangle$and $\left|5 / 2^{+}\right\rangle$levels.

\section{CONCLUSION}

The thorough study of the $A=35, T=3 / 2$ quartet shows a discrepancy from the accepted quadratic form of the IMME with a coefficient $d=-3.2(1.1) \mathrm{keV}$ for the cubic term. Questionable experimental data for excited ${ }^{35} \mathrm{Cl}$ levels have been found in the literature, and predictions based on isospin-mixing-dependent models indicate some possible deviation, too. Moreover, recent experimental data identified isospin-mixing effects in the vicinity of the $A=35, T=3 / 2$ quartet. From the theoretical calculations and experimental data a nonzero coefficient $d$ or higher terms are also possible. Further experimental investigations and a data recheck are needed to confirm this new "breakdown" of the IMME. For example, a direct measurement of the ${ }^{35} \mathrm{Ar}$ ground state should be performed with the Penning trap technique. Additional decay studies and spin-assignment checks for the ${ }^{35} \mathrm{Cl}$ and ${ }^{35} \mathrm{Ar}$ mirror nuclides should be performed to find new isospin-mixing effects for lower spin levels. Finally, new challenges are opened to theoretical calculations to reproduce the experimental data with better precision.

\section{ACKNOWLEDGMENTS}

The authors would like to thank A. Brown, A. García, and P. Van Isacker for fruitful discussions and the ISOLDE Collaboration and the technical team for their support. This work was supported by the German Ministry for Education and Research (BMBF) under Contract Nos. 06GF151 and 06MZ215 and the European Commission within the NIPNET RTD and EURONS/TRAPSPEC networks under Contract Nos. HPRICT-2001-50034 and RII3-CT-2004-506065, respectively. C.Y. was funded by the European Commission under the Marie Curie Fellowship network HPMT-CT-2000-00197. K.B. and S.G. are funded by the Helmholtz Association for National Research Centers (HGF) under Contract No. VH-NG-037.
[1] W. Heisenberg, Z. Phys. 77, 1 (1932).

[2] E. Wigner, Phys. Rev. 51, 106 (1937).

[3] E. P. Wigner, in The Robert A. Welch Foundation Conferences on Chemical Research. I. The structure of the Nucleus (Houston, Texas, November 20-22, 1957), edited by W. O. Milligan (Robert A. Welch Foundation, Houston, Texas, 1958), p. 67.

[4] S. Weinberg and S. B. Treiman, Phys. Rev. 116, 465 (1959).

[5] D. Lunney, J. M. Pearson, and C. Thibault, Rev. Mod. Phys. 75, 1021 (2003).

[6] H. Schatz, Int. J. Mass Spectrom. 251, 293 (2006).

[7] W. Benenson and E. Kashy, Rev. Mod. Phys. 51, 527 (1979).

[8] F. Herfurth, J. Dilling, A. Kellerbauer, G. Audi, D. Beck, G. Bollen, H.-J. Kluge, D. Lunney, R. B. Moore, C. Scheidenberger, S. Schwarz, G. Sikler, J. Szerypo, and the ISOLDE Collaboration, Phys. Rev. Lett. 87, 142501 (2001).

[9] M. C. Pyle, A. García, E. Tatar, J. Cox, B. K. Nayak, S. Triambak, B. Laughman, A. Komives, L. O. Lamm, J. E. Rolon, T. Finnessy, L. D. Knutson, and P. A. Voytas, Phys. Rev. Lett. 88, 122501 (2002).

[10] K. Blaum, G. Audi, D. Beck, G. Bollen, F. Herfurth, A. Kellerbauer, H.-J. Kluge, E. Sauvan, and S. Schwarz, Phys. Rev. Lett. 91, 260801 (2003).

[11] J. Britz, A. Pape, and M. Antony, At. Data Nucl. Data Tables 69, 125 (1998).

[12] G. Bertsch and K. Kahana, Phys. Lett. B33, 193 (1970).

[13] G. Bollen, S. Becker, H. J. Kluge, M. König, R. B. Moore, T. Otto, H. Raimbault-Hartmann, G. Savard, L. Schweikhard,
H. Stolzenberg, and the ISOLDE Collaboration, Nucl. Instrum. Methods A 368, 675 (1996).

[14] E. Kugler, Hyperfine Interact. 129, 23 (2000).

[15] Special issue of the International Journal of Mass Spectrometry, edited by L. Schweikhard and G. Bollen (Elsevier, Amsterdam, 2006) Vol. 251/2-3, pp. 85-312.

[16] K. Blaum, Phys. Rep. 425, 1 (2006).

[17] A. Kellerbauer, K. Blaum, G. Bollen, F. Herfurth, H.-J. Kluge, M. Kuckein, E. Sauvan, C. Scheidenberger, and L. Schweikhard, Eur. Phys. J. D 22, 53 (2003).

[18] F. Herfurth, J. Dilling, A. Kellerbauer, G. Bollen, S. Henry, H.-J. Kluge, E. Lamour, D. Lunney, R. B. Moore, C. Scheidenberger, S. Schwarz, G. Sikler, and J. Szerypo, Nucl. Instrum. Methods A 469, 254 (2001).

[19] G. Savard, St. Becker, G. Bollen, H.-J. Kluge, R. B. Moore, Th. Otto, L. Schweikhard, H. Stolzenberg, and U. Wiess, Phys. Lett. A158, 247 (1991).

[20] K. Blaum, G. Bollen, F. Herfurth, A. Kellerbauer, H.-J. Kluge, M. Kuckein, S. Heinz, P. Schmidt, and L. Schweikhard, J. Phys. B 36, 921 (2003).

[21] G. Gräff, H. Kalinowsky, and J. Traut, Z. Phys. A 297, 35 (1980).

[22] C. Yazidjian, K. Blaum, R. Ferrer, F. Herfurth, A. Herlert, and L. Schweikhard, Hyperfine Interact., in print.

[23] M. König, G. Bollen, H.-J. Kluge, T. Otto, and J. Szerypo, Int. J. Mass Spectrom. Ion Process. 142, 95 (1995).

[24] G. Audi, A. H. Wapstra, and C. Thibault, Nucl. Phys. A729, 337 (2003). 
[25] NNDC, Brookhaven National Laboratory, http://www.nndc. bnl.gov/masses/mass.mas03.

[26] A. H. Wapstra, G. Audi, and C. Thibault, Nucl. Phys. A729, 129 (2003).

[27] D. R. Goosman, K. W. Jones, E. K. Warburton, and D. E. Alburger, Phys. Rev. C 4, 1800 (1971).

[28] J. M. Freeman, Nucl. Instrum. Methods 134, 153 (1976).

[29] A. A. Jaffe, G. A. Bissinger, S. M. Shafroth, T. A. White, T. G. Dzubay, F. Everling, D. W. Miller, and D. A. Outlaw, Phys. Rev. C 3, 2489 (1971).

[30] T. G. Dzubay, A. A. Jaffe, E. J. Ludwig, T. A. White, F. Everling, D. W. Miller, and D. A. Outlaw, Phys. Lett. B33, 302 (1971).

[31] P. M. Endt, Nucl. Phys. A521, 1 (1990).

[32] U. Köster, Nucl. Phys. A701, 441 (2002).

[33] K. Blaum, D. Beck, G. Bollen, P. Delahaye, C. Guénaut, F. Herfurth, A. Kellerbauer, H.-J. Kluge, D. Lunney, S. Schwarz, L. Schweikhard, and C. Yazidjian, Europhys. Lett. 67, 586 (2004).

[34] J. Van Roosbroeck, C. Guénaut, G. Audi, D. Beck, K. Blaum, G. Bollen, J. Cederkall, P. Delahaye, A. De Maesschalck, H. De Witte, D. Fedorov, V. N. Fedoseyev, S. Franchoo, H. O. U. Fynbo, M. Górska, F. Herfurth, K. Heyde, M. Huyse, A. Kellerbauer, H.-J. Kluge, U. Köster, K. Kruglov, D. Lunney, V. I. Mishin, W. F. Mueller, Sz. Nagy, S. Schwarz, L. Schweikhard, N. A. Smirnova, K. Van de Vel, P. Van Duppen, A. Van Dyck, W. B. Walters, L. Weissman, and C. Yazidjian, Phys. Rev. Lett. 92, 112501 (2004).

[35] T. Lindqvist and A. C. G. Mitchell, Phys. Rev. 95, 444 (1954).

[36] N. Benczer-Koller, A. Schwarzschild, and C. S. Wu, Phys. Rev. 115, 108 (1959).

[37] F. Ajzenberg-Selove and G. Igo, Nucl. Phys. A142, 641 (1970).

[38] V. N. Levkovskii and I. V. Kazachevskii, Yadernaya Fizika 11, 483 (1970) [Sov. J. Nucl. Phys. 11, 271 (1970)].

[39] R. Santo, R. Stock, J. H. Bjerregaad, O. Hansen, O. Nathan, R. Chapman, and S. Hinds, Nucl. Phys. A118, 409 (1968).

[40] A. Marinov and J. R. Erskine, Phys. Lett. 14, 46 (1965).

[41] P. M. Endt, Nucl. Phys. A633, 1 (1998).

[42] A. García, E. G. Adelberger, P. V. Magnus, H. E Swanson, F. E. Wietfeldt, O. Tengblad, and the ISOLDE Collaboration, Phys. Rev. C 51, 3487 (1995).
[43] J. M. Freeman, R. J. Petty, S. H. Hoath, J. S. Ryder, W. E. Burcham, and G. T. A. Squier, in Proceedings of the Fifth International Conference on Atomic Masses and Fundamental Constants (Paris, June, 1975), edited by J. H. Sandars and A. H. Wapstra (Plenum Press, New York, 1976), p. 126.

[44] R. E. White and H. Naylor, Aust. J. Phys. 30, 365 (1977).

[45] G. Azuelos, G. R. Rao, and P. Taras, Phys. Rev. C 17, 443 (1978).

[46] L. G. Smith, Phys. Rev. C 4, 22 (1971).

[47] T. Altzitzoglou, F. Calaprice, M. Dewey, M. Lowry, L. Piilonen, J. Brorson, S. Hagen, and F. Loeser, Phys. Rev. Lett. 55, 799 (1985).

[48] T. Ohi, M. Nakajima, H. Tamura, T. Matsuzaki, T. Yamazaki, O. Hashimoto, and R. S. Hayano, Phys. Lett. B160, 322 (1985).

[49] J. J. Simpson and A. Hime, Phys. Rev. D 39, 1825 (1989).

[50] M. Chen, D. A. Imel, T. J. Radcliffe, H. Henrikson, and F. Boehm, Phys. Rev. Lett. 69, 3151 (1992).

[51] G. E. Berman, M. L. Pitt, F. P. Calaprice, and M. M. Lowry, Phys. Rev. C 48, R1 (1993).

[52] J. L. Mortara, I. Ahmad, K. P. Coulter, S. J. Freedman, B. K. Fujikawa, J. P. Greene, J. P. Schiffer, W. H. Trzaska, and A. R. Zeuli, Phys. Rev. Lett. 70, 394 (1993).

[53] P. Hubert, M. M. Aleonard, D. Castera, F. Leccia, and P. Mennrath, Nucl. Phys. A195, 485 (1972).

[54] D. D. Watson, J. C. Manthuruthill, and F. D. Lee, Phys. Rev. 164, 1399 (1972).

[55] A. Graue, L. H. Herland, J. R. Lien, G. E. Sandvik, E. R. Cosman, and W. H. Moore, Nucl. Phys. A136, 577 (1969).

[56] B. Fant, J. Keinonen, A. Anttila, and M. Bister, Z. Physik 260, 185 (1973).

[57] M. A. Meyer, I. Venter, W. F. Coetzee, and D. Reitmann, Nucl. Phys. A264, 13 (1976).

[58] B. A. Brown and B. H. Wildenthal, Annu. Rev. Nucl. Part. Sci. 38, 29 (1988).

[59] W. E. Ormand and B. A. Brown, Nucl. Phys. A491, 1 (1989).

[60] E. M. Henley and C. E. Lacy, Phys. Rev. 184, 1228 (1969).

[61] J. Ekman, D. Rudolph, C. Fahlander, A. P. Zuker, M. A. Bentley, S. M. Lenzi, C. Andreoiu, M. Axiotis, G. de Angelis, E. Farnea, A. Gadea, Th. Kröll, N. Marginean, T. Martinez, M. N. Mineva, C. Rossi-Alvarez, and C. A. Ur, Phys. Rev. Lett. 92, 132502 (2004). 